\title{
X-Band Outphasing Power Amplifier with Internal Load Modulation Measurements
}

\author{
Michael Litchfield, Tibault Reveyrand and Zoya Popović \\ Department of Electrical, Computer, and Energy Engineering (ECEE) \\ University of Colorado at Boulder, CO 80309-0425, USA
}

\begin{abstract}
This paper presents a general measurement based design method for outphasing amplifiers. Both isolated and nonisolated combiners are designed based on individual PA loadpull measurements, in order to load modulate two $0.15 \mu \mathrm{m}$ GaN MMIC power amplifiers at $10.1 \mathrm{GHz}$. Load modulation measurements are performed at ports internal to the $\mathrm{PA}$, with the inclusion of couplers in the combiner and a four-port LSNA, to gain insight into the dynamics of load modulation during outphasing operation. Both outphasing systems exhibit a peak PAE greater than $41.5 \%$ at a peak output power greater than $35.7 \mathrm{dBm}$.
\end{abstract}

Keywords-Power Amplifiers, Outphasing, Load Modulation, GaN, LSNA.

\section{INTRODUCTION}

Architectures using multiple power amplifiers, such as Doherty and outphasing, are commonly used to maintain high power added efficiency (PAE) over a large output power range. An insightful analysis of internal PAs requires internal measurement with dedicated instrumentation. Conventional measurement techniques focus on either externally measurable parameters of the PA system, or measurement of static subsystems. Such measurements fall short of predicting subsystem performance in full system operation, especially under load modulation conditions. Multiple PA circuits can benefit from measurements at internal circuit nodes, such as the timedomain measurement of waveforms at the interstage of a PA with gate voltage shaping [1]. Individual performance of the main and peaking amplifiers in a Doherty PA were also reported using a non-contact near-field technique [2]. The input waveforms of a broadband Doherty-outphasing PA using a 2-port LSNA are used to determine input phase, while the output is only measured with a power meter in [3]. The results presented here further the understanding of the outphasing PA through internal circuit measurements at both input and output of the internal PAs during full outphasing operation. In particular, $v$ and $i$ measurements in the output ports of the individual PAs give insight into the dynamic load-pulling mechanism of a non-isolated outphasing PA.

An outphasing amplifier [4] consists of two high-efficiency PAs, each driven in parallel with constant input power, and an output power combiner that can be isolated or non-isolated. The differential phase of the input signal contains envelope information that is reconstructed at the output. In non-isolated outphasing PAs, load modulation is significant and varies the individual PA performances. Therefore, it is important to understand and verify experimentally the effect of load modulation on the efficiency and power of each PA. Analyses leading to the design of non-isolated combiners in [5]-[7]
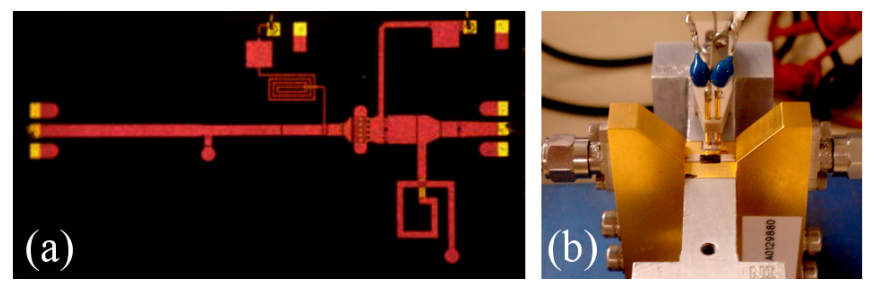

Fig. 1. The X-Band MMIC PA (a) used in this design is a single-stage PA using a $10 \times 100 \mu \mathrm{m}$ FET in TriQuint's $0.15 \mu \mathrm{m}$ GaN process. The chip is $3.8 \mathrm{~mm} \times 2.3 \mathrm{~mm}$. The MMIC is mounted in a $2.9 \mathrm{~mm}$ coaxial test-fixture (b), with internal measurements referenced to the RF pads of the MMIC. The test-fixture error terms are extracted from TRL calibration measurements.

reference the load modulation to the virtual drain of an ideal voltage source or ideal PA class, and focus on designing the combining power factor. In a practical design, the PA and combiner are often designed separately [8] and the load modulation should be designed to optimize performance by referencing it to the PA output. This paper presents a measurement-based method for designing X-Band outphasing power amplifiers. This method consists of two steps: first, the MMIC PAs are designed to maximize efficiency and characterized in large signal for load modulation design, then hybrid combiners are designed to maximize system performance with the given PAs. Both isolated and non-isolated combiners are designed based on the large signal characterization of individual PAs at $10.1 \mathrm{GHz}$. The dynamic load modulation between the internal PAs is measured to validate the design method. Finally, internal (individual PAs) and external system performances are measured and presented for both combiner topologies. To the best of the authors' knowledge, an outphasing PA has not been presented above $5 \mathrm{GHz}$ [9] and internal load modulation measurements have not been studied to date.

\section{MMIC PA DESIGN AND CHARACTERIZATION}

This work utilizes two Class-B GaN MMIC PAs fabricated in TriQuint's $0.15 \mu \mathrm{m}$ process, as described in [10] and shown in Fig. 1. To inform the design of the hybrid combiner (load modulation), the MMIC PAs are first characterized with loadpull measurements.

The large-signal measurement setup, seen in Fig. 2, is based on a large signal network analyzer (LSNA): the VTD SWAP $\mathrm{X}-402$ [11], which can measure the RF voltage and current at a defined reference plane after calibration. The system is calibrated with an absolute coaxial SOLT method [12]. The SWAP does not include any Harmonic Phase Reference (HPR). The absolute phase calibration is performed during the reverse "Thru" measurement by one of the LSNA receivers (seen 


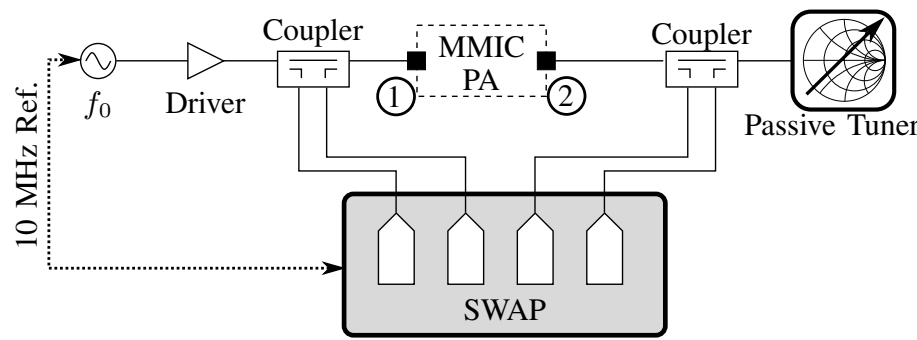

Fig. 2. The 2-port LSNA measurement setup for MMIC PA load-pull characterization. Measurement reference planes are de-embedded to the MMIC PA RF pads within the test-fixture.

as a RF scope), which is connected directly to the RF path as explained in [13]. After calibration, system measurements are associated with an error-term matrix defined in Eqn. (1), establishing a correction to apply at the measured RF frequency between the reference plane (1) and (2) in Fig. 2), measured quantities (RF voltages $v_{i}$ and current $i_{i}$ ), and the raw data acquired at the image frequency on the LSNA's ADCs (noted $r_{i}$ ).

$$
\left(\begin{array}{c}
v_{1} \\
i_{1} \\
v_{2} \\
i_{2}
\end{array}\right)=\left[\begin{array}{cccc}
\alpha_{1} & \beta_{1} & 0 & 0 \\
\gamma_{1} & \delta_{1} & 0 & 0 \\
0 & 0 & \alpha_{2} & \beta_{2} \\
0 & 0 & \gamma_{2} & \delta_{2}
\end{array}\right] \cdot\left(\begin{array}{l}
r_{1} \\
r_{2} \\
r_{3} \\
r_{4}
\end{array}\right)
$$

Each LSNA port is associated with a unique, 4-term error matrix. Internal measurements are de-embedded to the RF pads on the GaN MMIC PA, as seen in Fig. 1, using the test-fixture error terms extracted from a TRL calibration measurement.

Load-pull contours $\left(P A E\right.$ and $\left.P_{\text {out }}\right)$ are summarized in Fig. 3, along with designed load modulation contours $\left(\Gamma_{P A 1}\right.$ and $\left.\Gamma_{P A 2}\right)$ which are discussed in the next section. At a drain voltage of $20 \mathrm{~V}$, the measured peak power is $35.5 \mathrm{dBm}$ and the measured peak PAE is $67.1 \%$. A critical part of PA characterization for outphasing amplifier design is identifying the axis of impedances over which the power load-pull contours are symmetric. The load modulation $\left(\Gamma_{P A 1}\right.$ and $\left.\Gamma_{P A 2}\right)$ of the combiner is designed to be symmetric over this axis to ensure that each PA produces equal output power for stability and balance.

\section{OUtPhASING COMBINER DESIGNS}

In order to measure internally the individual PA performances and dynamic load modulation, it is necessary to include low-loss $(0.2 \mathrm{~dB})$ bi-directional couplers in the combiner design, as seen in Fig. 4(top). Both isolated and non-isolated combiners are designed, fabricated, and tested as follows.

\section{A. Non-Isolated Combiner}

The non-isolated combiner is shown in Fig. 4a and fabricated on 30 mil Rogers 4350B substrate. The design is based on a tee-junction topology and optimized by adding conjugate susceptances [4] and varying the lengths of the tee-junction lines around $90^{\circ}$ to adjust the load modulation [14]. An Sparameter analysis, at the fundamental frequency, on the nonisolated combiner yields equations useful for designing the

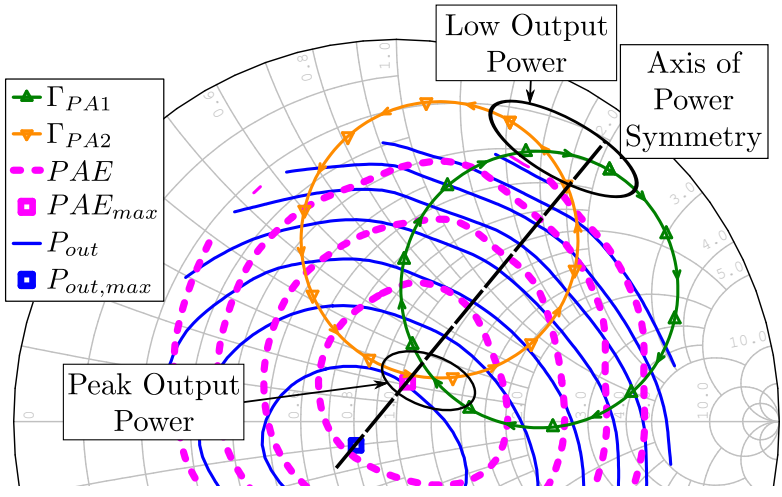

Fig. 3. Measured output power and PAE load-pull contours at a constant input power $\left(P_{i n}=26 \mathrm{dBm}\right)$ with axis of power symmetry shown for design, and load modulation predicted by Eqns. (3), (4) for non-isolated combiner. CW load-pull measurements have been performed with a LSNA (VTD-SWAP) and a passive tuner at $10.1 \mathrm{GHz}$. PAE contours are shown from $30 \%$ to $60 \%$ (with a $10 \%$ step) and $P_{\text {out }}$ contours are traced from 28 to $35 \mathrm{dBm}$ (with $1 \mathrm{~dB}$ step).

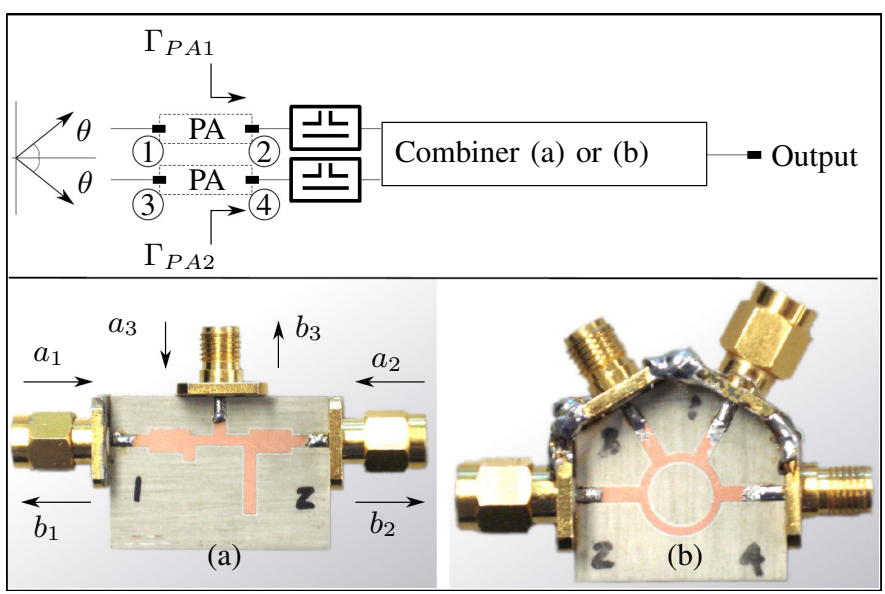

Fig. 4. Principle of outphasing PA illustrated on the top. The power combiner can be non-isolated (a) or isolated (b). The fabricated combiners are operating at $10.1 \mathrm{GHz}$, on $30 \mathrm{mil}$ Ro4350B substrate.

load modulation contours. Defining the input power waves shown in Fig. 4a as:

$$
a_{1}=x \cdot a_{2} e^{j \theta}, a_{2}=\frac{1}{x} \cdot a_{1} e^{-j \theta}, a_{3}=0
$$

where the port 3 is assumed to be matched. $x$ predicts the effect of PA output power imbalance, and $\theta$ is the swept differential phase between the branches. Equations for $b_{1}$ and $b_{2}$ can be solved for the reflection coefficient at each input port of the combiner, corresponding to the load modulation at the output of each individual PA, as:

$$
\begin{gathered}
\Gamma_{P A 1}=S_{11}+\frac{1}{x} \cdot S_{12} e^{-j \theta} \\
\Gamma_{P A 2}=S_{22}+x \cdot S_{21} e^{j \theta}
\end{gathered}
$$

The predicted load modulation is shown in Fig. 3. Because the load-pull characterization is performed at the MMIC reference plane, the design of the load modulation should occur at this plane as well. All transitions (including test-fixture) 


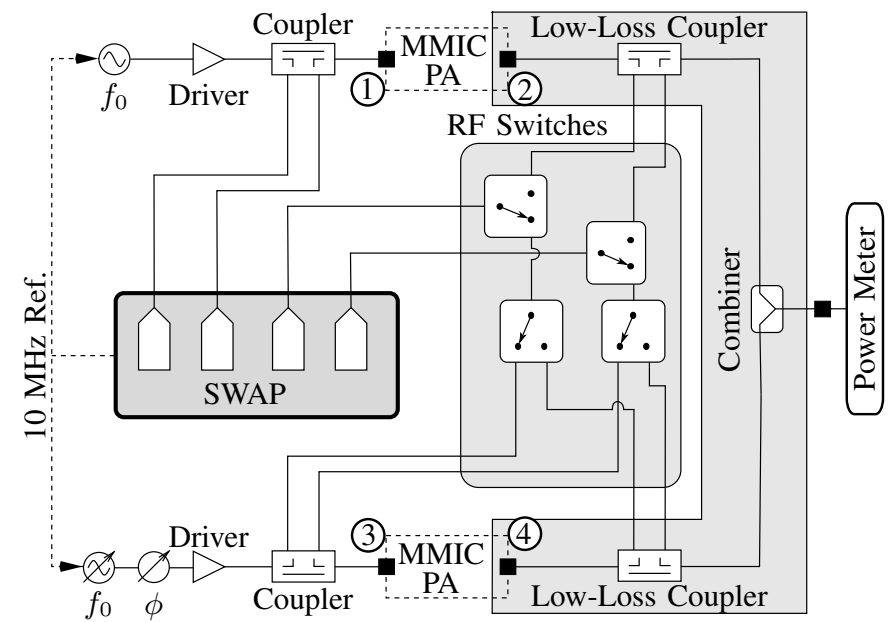

Fig. 5. The measurement setup is based on a 4-port LSNA. The two output couplers are included in the combiner, enabling internal load modulation measurements. A phase shifter $(\phi)$ sweeps the differential phase at an input power level which saturates both PAs.

and couplers on the output of the MMIC are measured and included in the combiner design. The design goal is to intersect the load modulation contours at the measured peak efficiency and remain in the highest efficiency impedance region possible, while maintaining symmetry over the axis of power symmetry. This design reduces the output power in order to ensure the PAs are loaded for highest possible efficiency near peak output power.

\section{B. Isolated Combiner}

The isolated combiner, a standard rat-race circuit depicted in Fig. $4 \mathrm{~b}$, provides a $180^{\circ}$ phase shift in order to sum and difference the signals from the parallel PAs. The loss between either PA input and either output is between 1.0 and $1.4 \mathrm{~dB}$, mostly due to the losses in the test-fixture and couplers. The return loss on both input ports is better than $19.5 \mathrm{~dB}$, and the isolation between the PA inputs is $22.5 \mathrm{~dB}$. The measured phase between paths to the summing output $\left(\angle S_{31}-\angle S_{32}\right)$ is $4.5^{\circ}$, while the phase between paths to the difference output $\left(\angle S_{41}-\angle S_{42}\right)$ is $173^{\circ}$.

\section{Internal LOAD Modulation MEAsurements}

The large-signal measurement setup presented in section II has been enhanced to 4-port capability with a switch-matrix consisting of four RF SPDTs as shown in Fig. 5. One of the four ports (noted (1)) is directly connected to two LSNA receiver channels, providing a phase reference for the three other ports, which are measured sequentially and are time domain-aligned to the first port.

The calibration of the system consists of performing three standard LSNA calibrations, one for each switch matrix position, in order to extract an error-term calibration matrix for every port as follows:

$$
\left(\begin{array}{c}
v_{i} \\
i_{i}
\end{array}\right)=\left[\begin{array}{ll}
\alpha_{i} & \beta_{i} \\
\gamma_{i} & \delta_{i}
\end{array}\right] \cdot\left(\begin{array}{l}
r_{3} \\
r_{4}
\end{array}\right)
$$

with $i=2,3$ and 4 for ports 2, 3, and 4 respectively. Each port is calibrated with the usual Short, Open, and Load standards, (a)

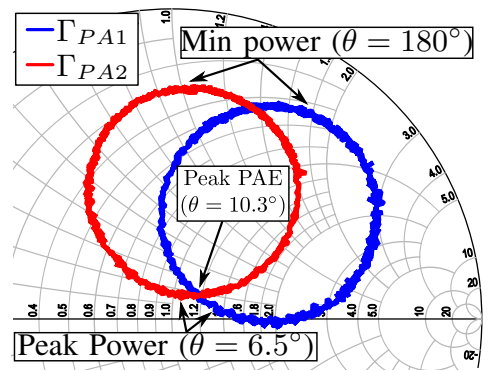

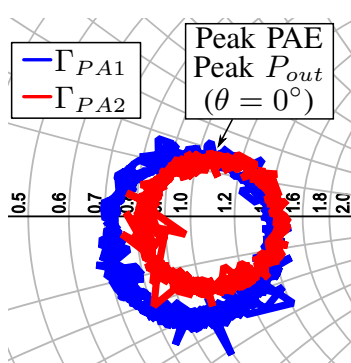

(b)
Fig. 6. Measurements of load modulation seen by internal PAs with nonisolated (a) and isolated (b) combiners. The load impedance seen by each PA is varied with the outphasing angle which changes the differential phase driving the parallel PAs.

before being connected with the Thru standard to the port 1 phase reference.

After calibration, the system measures RF voltage and current waveforms at the input and output of the two individual MMIC PAs. The complete outphasing PA system performance is measured simultaneously with a power meter at the output of the combiner as in [3]. During measurement, RF voltages and currents at ports 2,3 and 4 are acquired sequentially with a common port 1 measurement. A time domain alignment is performed by setting the phase of $v_{1}$ to $0^{\circ}$ at the fundamental frequency. Taking the input voltage as a phase reference is not an issue in a outphasing setup because the input power level remains constant and large.

A phase shifter $(\phi)$ sweeps the phase difference between the two branches. The second branch source amplitude is adjusted to maintain amplitude balance at the two inputs. Internal PA and load modulation measurements are de-embedded to the MMIC PA RF pads, shown in Fig. 1.

\section{A. Load Modulation Measurement Results}

For each phase point $\theta=\angle\left\{V_{\text {in } 2}\left(f_{0}\right) / V_{\text {in } 1}\left(f_{0}\right)\right\}$, the load impedance seen by each power amplifier was measured by the SWAP. This provides a measurement of the designed load modulation contours.

Fig. 6a shows the measured load seen by each PA as the phase between the two input voltages is swept from $-180^{\circ}$ to $180^{\circ}$. This measurement agrees with the designed load modulation shown in Fig. 3. The peak output power occurs near the crossing of the two load contours at an outphasing angle of $6.5^{\circ}$, due to their combined proximity to the peak power impedance. The peak PAE is measured at $\theta=10.3^{\circ}$. The minimum measured output power is $3.6 \mathrm{dBm}$, reached as the two load contours approach the edge of the Smith Chart.

Load modulation should not occur for the ideal isolated outphasing combiner with infinite isolation and $50 \Omega$ input impedances at the driven ports. Fig. $6 \mathrm{~b}$ shows the measured load modulation for the designed rat-race isolated combiner. The small load modulation circles are expected, because the input impedances at the driven ports are not exactly $50 \Omega$ at the MMIC reference plane and the isolation is finite. Furthermore, one load modulation circle is larger than the other because of a small internal PA output power imbalance. 


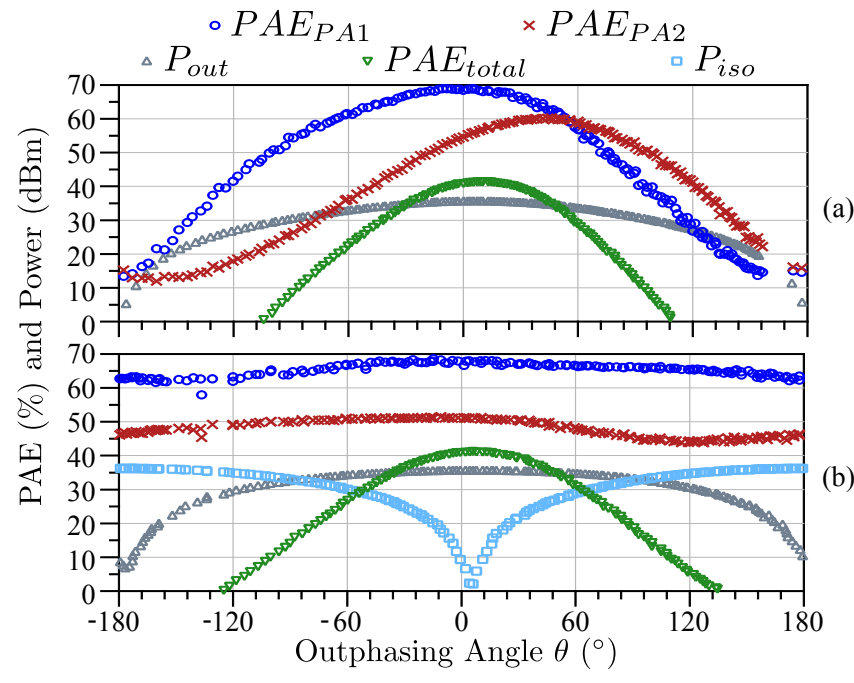

Fig. 7. Measured non-isolated (a) and isolated (b) outphasing system output powers and efficiencies and internal PA efficiencies. The non-isolated combiner shows a maximum $P A E$ of $41.5 \%$ at $\theta=10.3^{\circ}$ and a maximum $P_{\text {out }}$ of $35.7 \mathrm{dBm}$ at $\theta=6.5^{\circ}$. The isolated combiner shows a peak PAE of $41.6 \%$ at $\theta=8.2^{\circ}$ and a maximum $P_{\text {out }}$ of $35.8 \mathrm{dBm}$ at $\theta=3.7^{\circ}$.

\section{OutPhasing System Performance}

The system performance of the non-isolated outphasing PA is shown in Fig. 7a. The design achieves a peak output power of $35.7 \mathrm{dBm}$ with a peak PAE (PAE total $)$ of $41.5 \%$. This includes the loss in the couplers and transitions on the output. In an integrated design without couplers, the loss would reduce by $1.3 \mathrm{~dB}$, increasing the peak output power to $37 \mathrm{dBm}$ and the peak PAE to $60 \%$. The PAE of the internal PAs is shown in Fig. 7a: $P A E_{P A 1}$ and $P A E_{P A 2}$. Because the PAs are not ideal or balanced, maximum internal PAEs do not occur at the same outphasing angle as maximum system efficiency.

Fig. $7 \mathrm{~b}$ shows the system performance of the isolated outphasing amplifier. As expected, the system efficiency is almost exactly symmetric about the peak power which occurs very close to zero outphasing angle. The measured peak power is $35.8 \mathrm{dBm}$ and the peak PAE is $41.6 \%$. Again, losses in couplers and transitions are included, so integration would decrease the loss by $1 \mathrm{~dB}$, increasing the peak power to $36.8 \mathrm{~dB}$ and peak PAE to $59.1 \%$. Because the PAs only experience a small range of loading due to the combiner isolation, their individual PAEs are flat across outphasing angle. Thus the system PAE is most influenced by the system output power characteristic, which causes the PAE roll-off with power backoff level to be steep.

Finally, Fig. 8 compares the system efficiencies for both measured systems above. The PAE is plotted against the power back-off level, relevant to high PAR signals. Note that system drain efficiencies are $10 \%$ greater than PAEs, due to the use of compressed, single-stage individual PAs as well as the constant input power in the outphasing system.

\section{CONCLUSION}

A practical design methodology that treats both the PA and combiner is demonstrated through implementation at $10.1 \mathrm{GHz}$ with GaN MMIC PAs and hybrid combiners. The internal

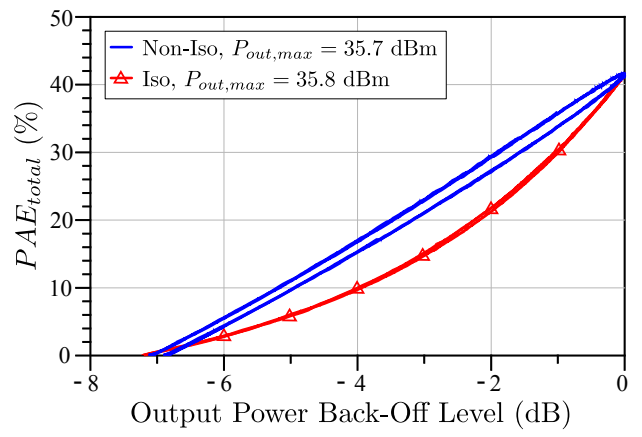

Fig. 8. Comparison of measured system PAE's for isolated and non-isolated outphasing.

load modulation is measured to confirm the design and give insight into internal behavior during dynamic operation. Due to care taken to balance the internal PA output powers, the measured load modulation is well-behaved and close to the expected design. Both isolated and non-isolated outphasing PAs are demonstrated with peak PAEs higher than $41 \%$ and peak output powers above $35.7 \mathrm{dBm}$ (PAEs higher than $59 \%$ and peak output powers above $5 \mathrm{~W}$ for integrated designs).

\section{REFERENCES}

[1] A. Ramadan et al., "Two-stage gan hemt amplifier with gate-source voltage shaping for efficiency versus bandwidth enhancements," IEEE Transactions on MTT, vol. 59, no. 3, pp. 699-706, 2011.

[2] R. Hou et al., "Non-intrusive characterization of active device interactions in high-efficiency power amplifiers," in IEEE MTT-S IMS Digest. IEEE, 2013, pp. 1-3.

[3] C. Andersson et al., "A 1-3-ghz digitally controlled dual-rf input poweramplifier design based on a doherty-outphasing continuum analysis," IEEE Transactions on MTT, vol. 61, no. 10, pp. 3743-3752, 2013.

[4] H. Chireix, "High power outphasing modulation," Radio Engineers, Proceedings of the Institute of, vol. 23, no. 11, pp. 1370-1392, 1935.

[5] F. Raab, "Efficiency of outphasing rf power-amplifier systems," IEEE Transactions on Communications, vol. 33, no. 10, pp. 1094-1099, 1985.

[6] M. El-Asmar et al., "Analytical design methodology of outphasing amplification systems using a new simplified chireix combiner model," IEEE Transactions on MTT, vol. 60, no. 6, pp. 1886-1895, 2012.

[7] B. Stengel and W. Eisenstadt, "Linc power amplifier combiner method efficiency optimization," Vehicular Technology, IEEE Transactions on, vol. 49, no. 1, pp. 229-234, 2000.

[8] D. Calvillo-Cortes et al., "A package-integrated chireix outphasing rf switch-mode high-power amplifier," IEEE Transactions on MTT, vol. 10, no. 61, pp. 3721 - 3732, 2013.

[9] J. Grundlingh, K. Parker, and G. Rabjohn, "A high efficiency Chireix out-phasing power amplifier for $5 \mathrm{GHz}$ WLAN applications," in IEEE MTT-S IMS Digest, vol. 3. IEEE, 2004, pp. 1535-1538.

[10] S. Schafer et al., "X-Band MMIC GaN Power Amplifiers Designed for High-Efficiency Supply-Modulated Transmitters," in IEEE MTT-S IMS Digest, Seattle, WA, 2013, pp. 1-4.

[11] P. Roblin, Nonlinear RF circuits and nonlinear vector network analyzers. Cambridge University Press, 2011.

[12] J. Verspecht, "Calibration of a measurement system for high frequency nonlinear devices," Brussels, Belgium Vrije Univeriteit Brussels, Ph. D. Thesis, 1995.

[13] K. Andersson and C. Fager, "Oscilloscope based two-port measurement system using error-corrected modulated signals," in Integrated Nonlinear Microwave and Millimetre-Wave Circuits (INMMIC). IEEE, 2012, pp. 1-3.

[14] W. Gerhard and R. Knoechel, "Improved design of outphasing power amplifier combiners," in German Microwave Conference, 2009, pp. 1-4. 